\title{
Efficacy of different types of aerobic exercise in fibromyalgia syndrome: a systematic review and meta-analysis of randomised controlled trials
}

\author{
Winfried Häuser*1,2, Petra Klose³, Jost Langhorst 3 , Babak Moradi4 ${ }^{4}$ Mario Steinbach4 ${ }^{4}$ Marcus Schiltenwolf4 and \\ Angela Busch 5
}

\begin{abstract}
Introduction: The efficacy and the optimal type and volume of aerobic exercise (AE) in fibromyalgia syndrome (FMS) are not established. We therefore assessed the efficacy of different types and volumes of AE in FMS.

Methods: The Cochrane Library, EMBASE, MEDLINE, PsychInfo and SPORTDISCUS (through April 2009) and the reference sections of original studies and systematic reviews on AE in FMS were systematically reviewed. Randomised controlled trials (RCTs) of AE compared with controls (treatment as usual, attention placebo, active therapy) and headto-head comparisons of different types of AE were included. Two authors independently extracted articles using predefined data fields, including study quality indicators.

Results: Twenty-eight RCTs comparing AE with controls and seven RCTs comparing different types of AE with a total of 2,494 patients were reviewed. Effects were summarised using standardised mean differences ( $95 \%$ confidence intervals) by random effect models. AE reduced pain $(-0.31(-0.46,-0.17) ; P<0.001)$, fatigue $(-0.22(-0.38,-0.05) ; P=$ $0.009)$, depressed mood $(-0.32(-0.53,-0.12) ; P=0.002)$ and limitations of health-related quality of life (HRQOL) $(-0.40(-$ $0.60,-0.20) ; P<0.001)$, and improved physical fitness $(0.65(0.38,0.95) ; P<0.001)$, post treatment. Pain was significantly reduced post treatment by land-based and water-based $A E$, exercises with slight to moderate intensity and frequency of two or three times per week. Positive effects on depressed mood, HRQOL and physical fitness could be maintained at follow-up. Continuing exercise was associated with positive outcomes at follow-up. Risks of bias analyses did not change the robustness of the results. Few studies reported a detailed exercise protocol, thus limiting subgroup analyses of different types of exercise.

Conclusions: An aerobic exercise programme for FMS patients should consist of land-based or water-based exercises with slight to moderate intensity two or three times per week for at least 4 weeks. The patient should be motivated to continue exercise after participating in an exercise programme.
\end{abstract}

\section{Introduction}

The key symptoms of fibromyalgia syndrome (FMS) are chronic widespread (both sides, above and below waist line, and axial skeletal) pain, fatigue, sleep disturbances and tenderness on palpation [1]. The estimated prevalence of FMS in western countries ranges from 2.2 to $6.6 \%[2]$. Comorbidities with other functional somatic syndromes and mental disorders are common [3]. FMS is

* Correspondence: whaeuser@klinikum-saarbruecken.de

1 Department of Internal Medicine I, Klinikum Saarbrücken, Winterberg 1, D-66119 Saarbrücken, Germany

Full list of author information is available at the end of the article associated with high utilisation and costs of health services. Effective treatment options are therefore needed for medical and economic reasons [4].

Systematic reviews and evidence-based guidelines provide healthcare professionals and patients with a guide through the great variety of pharmacological and nonpharmacological treatment options in FMS. Three evidence-based guidelines available on the management gave different grades of recommendation for aerobic exercises (AE) (aerobic exercise with and without additional strength and flexibility training) in FMS. The 
American Pain Society [5] and the guidelines of the Association of the Scientific Medical Societies in Germany [6] gave the highest grade of recommendation for AE. The European League Against Rheumatism judged the published evidence for the efficacy of AE to be lacking [7]. Qualitative reviews on the efficacy of AE in FMS that searched the literature until December 2006 came to different conclusions on the short-term and long-term efficacy of AE in FMS [8-10].

More recently, Jones and Lipton reviewed over 70 FMS exercise studies and found similar results when protocols included yoga, tai chi and other movement-based therapies [11]. Two meta-analyses on exercise in FMS have been conducted. Busch and colleagues searched the literature until July 2005. Owing to significant clinical heterogeneity among the studies, only six studies with $\mathrm{AE}$ were meta-analysed. Moderate quality evidence was found that $\mathrm{AE}$ had positive effects on global well-being and physical function, but not on pain at post treatment [12]. The Ottawa Panel searched the literature until December 2006 and found most improvements for pain relief and increase of endurance at post treatment [13]. Outcomes at follow-up were not meta-analysed.

Not only the question of efficacy but also that of the dose and type of AE need to be clarified. The American Pain Society recommended encouraging patients to perform moderately intense $\mathrm{AE}$ (60 to $70 \%$ of age-adjusted predicted maximum heart rate (maxHR)) two or three times per week [5]. The evidence of this recommendation has not been tested by meta-analyses of head-to-head comparisons of different types and volumes of AE. Moreover, the question of whether continuing $\mathrm{AE}$ is required to maintain a symptom reduction had not been systematically addressed.

The aims of the present systematic review were to update the literature on AE in FMS and to assess whether $\mathrm{AE}$ has beneficial effects at post treatment and at followup on the key domains of FMS (pain, sleep, fatigue, depressed mood), compared with other therapies. In contrast to the Cochrane review [12], we intended to metaanalyse the outcomes of all randomised controlled trials (RCTs) available. Another aim was to asses which types, volumes and intensities of $\mathrm{AE}$ are effective by performing head-to-head comparisons of RCTs with different types and intensities of AE. The final aim was to assess whether ongoing exercise is necessary to maintain potential positive effects of AE.

\section{Materials and methods}

The present review was performed according to the Preferred Reporting Items for Systematic Reviews and MetaAnalyses statement [14] and the recommendations of the Cochrane Collaboration [15].

\section{Protocol}

Methods of analysis and inclusion criteria were specified in advance. We used the review protocol of our systematic review on multicomponent therapy in FMS [16].

\section{Eligibility criteria \\ Types of studies}

A RCT design comparing $\mathrm{AE}$ with a control group receiving no treatment, treatment as usual, attention control or any pharmacological or nonpharmacological therapy, or with head-to-head comparisons of different types or intensities of AE were included. Studies without randomisation were excluded.

\section{Types of participants}

Patients of any age diagnosed with FMS on recognised criteria were included.

\section{Types of intervention}

$\mathrm{AE}$ was assumed if the reported target heart rate of the training protocol was at least (on average) $40 \%$ of maxHR or if the training protocol included exercise involving at least one-sixth of the skeletal muscles (for example, walking, running, biking, aerobics, vibrations). At least $50 \%$ of the training session should consist of AE. In the case of mixed exercise, defined as a combination of $\mathrm{AE}$ with stretching and/or muscle strength [17], the length of $\mathrm{AE}$ should exceed the time with other types of exercise. Stretching during warm-up and cool-down periods was not defined as mixed exercise. No restrictions on frequency or duration of training were made.

We excluded studies or study arms in which AE was part of multicomponent therapy defined as a combination of $\mathrm{AE}$ with psychological therapy (structured education or relaxation therapy, cognitive-behavioural therapy) [16]. We excluded studies or study arms with balneotherapy (warm-water treatment without exercise).

\section{Types of outcomes measures}

Studies should assess at least one key domain of FMS (pain, sleep, fatigue, depressed mood and health-related quality of life (HRQOL)) (primary outcome measures). Secondary outcome measures were any measure of physical fitness.

\section{Data sources and searches}

The electronic bibliographic databases screened included the Cochrane Central Register of Controlled Trials (CENTRAL), EMBASE, MEDLINE, PsychInfo and SPORTDISCUS (through 31 March 2009). The search strategy for MEDLINE is detailed in Additional file 1. The search strategy was adapted for each database as necessary. No language restrictions were made. Only fully published papers were reviewed. In addition, reference sections of original studies, systematic reviews [8-10] and evidencebased guidelines on the management of FMS [4-6] were screened manually. 


\section{Study selection}

The search was conducted by two authors (PK, JL). Two authors screened the titles and the abstracts of potentially eligible studies identified by the search strategy detailed above independently (PK, JL). The full-text articles were then examined independently by two authors to determine whether they met the selection criteria (MSc, JL). Discrepancies were rechecked and consensus was achieved by discussion. If needed, two other authors reviewed the data to reach a consensus $(\mathrm{AB}, \mathrm{WH})$.

\section{Data collection process}

Two authors independently extracted the data using standard extraction forms [16] (BM, MSc). Discrepancies were rechecked and consensus was achieved by discussion. If needed, a third author reviewed the data to reach a consensus (WH).

Based on our experiences of former systematic reviews in which none of the contacted authors provided these details on request, we did not ask for clarifications of study design in case of unclear randomisation, blinding or concealment of treatment allocation. We searched for further details of the study design in a Cochrane review [12].

When means or standard deviations (SDs) were missing, attempts were made to obtain these data through contacting 12 trial authors. Additional data were provided by four authors (see Tables 1 and 2). Where SDs were not available from the trial authors, they were calculated from $t$ values, confidence intervals or standard errors when reported in articles [15]. If only the median was given, the median was used instead of the mean and a SD was substituted that was calculated as the mean of the SDs available for studies that used the same outcome scale.

\section{Data items}

The data for the study setting, participants, exclusion criteria, interventions, co-therapies, attendance rates, side effects reported and outcomes sought are presented in Tables 2 and 3.

When researchers reported more than one measure for an outcome, we used a predefined order of preference for analysis (details available on request).

If studies had two or more potential control groups, we used the following order to select for control group: treatment as usual, attention placebo, and active control to select the control group.

\section{Risk of bias in individual studies}

To ascertain the internal and external validity of the eligible RCTs, two pairs of reviewers (BM, WH; and MSc, Mst) working independently and with adequate reliability determined the adequacy of randomisation, concealment of allocation, blinding of outcome assessors and adequacy of data analysis (was intention-to-treat-analysis performed?) (internal validity). Furthermore we chose the item 'Were patients with mental disorders frequently associated with FMS (depressive and anxiety disorders) included in the studies?' as the marker of external validity.

\section{Summary measures}

Meta-analyses were conducted using RevMan Analyses software (RevMan 5.0.17) from the Cochrane collaboration [18]. Standardised mean differences (SMDs) were calculated by means and SDs or change scores for each intervention. The SMD used in Cochrane reviews is the effect size known as Hedge's (adjusted) $g$ [15]. Examination of the combined results was performed by a random effects model (inverse variance method), because this model is more conservative than the fixed effects model and incorporates both within-study and between-study variance [19]. We used Cohen's categories to evaluate the magnitude of the effect size, calculated by the SMD: $g>$ 0.2 to 0.5 , small effect size; $g>0.5$ to 0.8 , medium effect size; $g>0.8$, large effect size [20].

\section{Planned methods of analysis}

Heterogeneity was tested using the $I^{2}$ statistic, with $I^{2}>$ $50 \%$ indicating strong heterogeneity. $\tau^{2}$ was used to determine how much heterogeneity was explained by subgroup differences [15].

\section{Risk of bias across studies}

Potential publication bias - that is, the association of publication probability with the statistical significance of study results - was investigated using visual assessment of the funnel plot (plots of effect estimates against its standard error) calculated by RevMan Analyses software. Publication bias may lead to asymmetrical funnel plots [15]. Moreover, we checked a potential small sample size bias by a sensitivity analysis of studies with very small $(<25)$, small (25 to 50$)$ and medium $(>50)$ sample sizes.

\section{Additional analyses \\ Subgroup analysis}

The following subgroup analyses were pre-specified: types of AE (land-based, water-based and mixed; AE as monotherapy or combined with flexibility and/or strength), intensity of $\mathrm{AE}$ (very low intensity, $<50 \%$ of maxHR; low intensity, 50 to $60 \%$ of maxHR; moderate intensity, 60 to $80 \%$ maxHR; intensity left up to patient), frequency of AE per week (1 time/week, 2 times/week, 3 times/week and $>3$ times/week), duration of the study ( $<7$ weeks, 7 to 12 weeks, $>12$ weeks) and duration of total aerobic exercise ( $<1,000$ minutes, 1,000 to 2,000 minutes, $>2,000$ minutes), and type of control group (attention placebo, treatment as usual, other active therapy). These subgroup analyses were also used to examine potential sources of clinical heterogeneity. 
Table 1: Risk of bias (internal and external validity) of the randomised controlled trials' analysis

\begin{tabular}{|c|c|c|c|c|c|}
\hline Author, year & $\begin{array}{l}\text { Adequate } \\
\text { randomisation }\end{array}$ & $\begin{array}{l}\text { Adequate } \\
\text { allocation } \\
\text { concealment }\end{array}$ & $\begin{array}{l}\text { Blinding of } \\
\text { assessor }\end{array}$ & $\begin{array}{l}\text { Intention-to-treat } \\
\text { analysis }\end{array}$ & $\begin{array}{l}\text { Inclusion of } \\
\text { patients with } \\
\text { mental disorders }\end{array}$ \\
\hline Alentorn, 2008 & 0 & 0 & + & - & + \\
\hline Altan, 2004 & 0 & 0 & 0 & - & - \\
\hline Assis, 2006 & + & + & - & + & - \\
\hline Bircan, 2006 & 0 & 0 & 0 & - & + \\
\hline Buckelew, 2008 & 0 & 0 & 0 & - & + \\
\hline Da Costa, 2005 & + & + & + & + & + \\
\hline Ecvik, 2008 & 0 & 0 & 0 & - & + \\
\hline Etnier, 2009 & 0 & 0 & 0 & - & + \\
\hline Fontaine, 2007 & 0 & 0 & 0 & - & + \\
\hline Gowans, 2001 & 0 & 0 & 0 & - & + \\
\hline Gusi, 2006 & 0 & 0 & 0 & - & - \\
\hline Jentoft, 2001 & 0 & 0 & - & + & + \\
\hline Jones, 2008 & + & + & + & + & - \\
\hline King, 2002 & + & 0 & + & + & + \\
\hline Martin, 1996 & 0 & 0 & + & + & + \\
\hline McCain, 1988 & 0 & 0 & + & - & + \\
\hline Mengshoel, 1992 & + & 0 & 0 & + & + \\
\hline Meyer, 2000 & 0 & 0 & 0 & - & + \\
\hline Munguia, 2008 & + & + & + & + & - \\
\hline Nichols, 1994 & 0 & 0 & 0 & - & + \\
\hline Noregaard, 1997 & 0 & 0 & 0 & + & + \\
\hline Ramsay, 2000 & 0 & 0 & + & + & 0 \\
\hline Redondo, 2004 & + & 0 & 0 & + & - \\
\hline Richards, 2002 & + & + & + & + & + \\
\hline Rooks, 2007 & + & + & + & + & 0 \\
\hline Schachter, 2003 & + & + & + & + & + \\
\hline Sencan, 2004 & 0 & 0 & 0 & + & 0 \\
\hline Stephens, 2008 & + & + & + & + & + \\
\hline $\begin{array}{l}\text { Tomas-Carus, } \\
2008\end{array}$ & 0 & + & + & + & 0 \\
\hline Valim, 2003 & 0 & 0 & + & + & + \\
\hline Valkeinen, 2008 & + & 0 & 0 & + & 0 \\
\hline Van Santen, 2001 & 0 & - & - & - & 0 \\
\hline Van Santen, 2002 & 0 & 0 & + & + & - \\
\hline Vitorino, 2006 & + & + & - & + & - \\
\hline Wigers, 1996 & 0 & 0 & + & + & 0 \\
\hline
\end{tabular}

+ , yes; 0 , unclear; -, no. 
Table 2: Effect sizes of aerobic and mixed exercise on selected outcome variables

\begin{tabular}{|c|c|c|c|c|c|}
\hline Outcome title & $\begin{array}{l}\text { Number of } \\
\text { study arms }\end{array}$ & $\begin{array}{l}\text { Number of } \\
\text { patients on } \\
\text { aerobic exercise }\end{array}$ & Effect size $^{a}$ & $\begin{array}{l}\text { Test for overall } \\
\text { effect } P \text { value }\end{array}$ & $\begin{array}{l}\text { Heterogeneity, 12; } \\
\tau 2(\%)\end{array}$ \\
\hline \multicolumn{6}{|l|}{ Post treatment } \\
\hline 01 Pain & 29 & 567 & $-0.31(-0.46,-0.17)$ & $<0.001$ & $26 ; 0.03$ \\
\hline 02 Fatigue & 16 & 364 & $-0.22(-0.38,-0.05)$ & 0.009 & $9 ; 0.01$ \\
\hline 03 Sleep & 9 & 184 & $0.01(-0.19,0.21)$ & 0.92 & $0 ; 0$ \\
\hline $\begin{array}{l}\text { 04Depressed } \\
\text { mood }\end{array}$ & 19 & 456 & $-0.32(-0.53,-0.12)$ & 0.002 & $51 ; 0.10$ \\
\hline $05 \mathrm{HRQOL}$ & 25 & 526 & $-0.40(-0.60,-0.20)$ & $<0.001$ & $63 / 0.15$ \\
\hline $\begin{array}{l}06 \text { Physical } \\
\text { fitness }\end{array}$ & 20 & 339 & $0.65(0.38,0.93)$ & $<0.001$ & $71 / 0.20$ \\
\hline \multicolumn{6}{|l|}{ Latest follow-up } \\
\hline 01 Pain & 9 & 187 & $-0.13(-0.80,0.54)$ & 0.08 & $0 / 0$ \\
\hline 02 Fatigue & 4 & 93 & $-0.23(-0.62,0.17)$ & 0.26 & $42 / 0.07$ \\
\hline 03 Sleep & 4 & 84 & $0.17(-0.14,0.47)$ & 0.28 & $0 / 0$ \\
\hline $\begin{array}{l}\text { 04Depressed } \\
\text { mood }\end{array}$ & 8 & 151 & $-0.44(-0.88,0.01)$ & 0.05 & $71 / 0.22$ \\
\hline $05 \mathrm{HRQOL}$ & 8 & 221 & $-0.27(-0.48,-0.05)$ & 0.02 & $14 / 0.01$ \\
\hline $\begin{array}{l}06 \text { Physical } \\
\text { fitness }\end{array}$ & 5 & 99 & $0.65(0.35,0.96)$ & $<0.001$ & $0 / 0$ \\
\hline
\end{tabular}

HRQOL: health-related quality of life. aStandardised mean difference (95\% confidence interval).

\section{Sensitivity analyses}

The following sensitivity analyses were pre-specified: inadequate or unclear versus adequate sequence generation; inadequate or unclear allocation versus adequate concealment; intention-to-treat analysis, no versus yes; studies that provided medians of outcomes versus means of outcomes; and patients with mental disorders frequently associated with FMS excluded (yes or unclear).
These sensitivity analyses were also used to examine potential sources of methodological heterogeneity.

\section{Results}

\section{Study selection}

The literature search produced 464 citations, of which 292 were double hits (study found in at least two data sources). By screening, 110 records were excluded: 23 evaluated AE, but not in FMS; 19 did not evaluate AE in

Table 3: Effect sizes of head-to-head comparisons of different types of aerobic exercise on selected outcome variables

\begin{tabular}{|c|c|c|c|c|c|}
\hline $\begin{array}{l}\text { Outcome title } \\
\text { post treatment }\end{array}$ & $\begin{array}{l}\text { Number of } \\
\text { studies }\end{array}$ & $\begin{array}{l}\text { Number of } \\
\text { patients }\end{array}$ & Effect size ${ }^{a}$ & $\begin{array}{l}\text { Test for overall } \\
\text { effect, } P \text { value }\end{array}$ & $\begin{array}{l}\text { Heterogeneity, I2; } \\
\tau 2(\%)\end{array}$ \\
\hline \multicolumn{6}{|c|}{ Moderate intensity versus low intensity } \\
\hline 01 Pain & 2 & 68 & $-0.08(-1.41,1.26)$ & 0.91 & $78 ; 0.96$ \\
\hline $\begin{array}{l}\text { 02 Depressed } \\
\text { mood }\end{array}$ & 2 & 68 & $-0.16(-0.67,0.13)$ & 0.53 & $0 ; 0$ \\
\hline $\begin{array}{l}03 \text { Physical } \\
\text { fitness }\end{array}$ & 2 & 68 & $0.25(-0.26,0.75)$ & 0.34 & $0 ; 0$ \\
\hline \multicolumn{6}{|c|}{ Land-based versus water based exercise } \\
\hline 01 Pain & 9 & 187 & $-0.13(-0.80,0.54)$ & 0.08 & $0 / 0$ \\
\hline $\begin{array}{l}\text { 02 Depressed } \\
\text { mood }\end{array}$ & 8 & 151 & $-0.44(-0.88,0.01)$ & 0.05 & $71 / 0.22$ \\
\hline
\end{tabular}

aStandardised mean difference (95\% confidence interval). 
FMS; 52 were review articles; and 18 were case reports or commentaries. Sixty of the full-text articles assessed for eligibility, and 25 full-text articles were excluded for the following reasons: two for publication of different outcomes of one trial in two publications [21,22]; six for lacking a control group [23-28]; three for lacking randomisation [29-31]; two because one could not conclude from the study protocol that the exercises performed met the predefined criteria of $\mathrm{AE}[32,33]$; one because two different types of water-based exercise with similar intensity were compared [34]; one because the study did not assess a primary outcome measure [35]; and 10 because $\mathrm{AE}$ was combined with education or psychotherapy or pharmacotherapy [36-45]. Three RCTs comparing different intensities of $\mathrm{AE}$ [46-48], four RCTs comparing land-based with water-based exercise [49-52] and 28 RCTs with 29 study arms comparing AE with controls [53-80] were included in the qualitative and quantitative analyses (see Figure 1).

\section{Study characteristics \\ Setting, referral and exclusion criteria (representativeness of study samples)}

Fourteen studies each were conducted in North America, 13 studies in Europe and four studies each in South America (Brazil) and Asia (Turkey) (see Additional files 1, 2 and 3). Patients were recruited by register of hospitals, referral (general practitioner, rheumatologist, hospital departments), local self-help groups and newspaper advertisement. Thirty-two studies were conducted within the setting of a university, three within district hospitals. All studies were single-centre based. One study had two AE study arms.

Thirty-one studies excluded patients with internal diseases or with orthopaedic diseases precluding AE. Sixteen studies excluded patients with mental disorders including depression. Four studies excluded patients with unresolved litigation. No study reported comorbidities of the patients.

\section{Participants}

The median of the mean age of the participants was 45 years (13 to 59 years). One study included only children and adolescents. The median of the percentage of women was $100 \%$ (71 to $100 \%$ ).

\section{Interventions}

AE was supervised by a trainer in 32 studies. AE included cycling, walking, aquatic jogging, games, dance and rhythmic or boxing movements. Aerobic intensity was reported in 27 studies as a target heart rate or percentage age-predicted maxHR determined by standard equations. Percentage maxHRs were usually progressive and ranged from 40 to $80 \%$ of the age-predicted maximum. The target heart rate of 21 studies was between low and moderate intensity (50 to $80 \%$ ). Only one study prescribed a very low intensity (maxHR 30 to $50 \%$ ), and three studies recommended that patients should exercise with a moderate intensity subjectively determined by the patient without measuring the heart rate. Three studies did not report the recommended intensity.

Sixteen studies reported the attrition rates, with a median of $67 \%$ (range 27 to $90 \%$ ).

In 12 studies the controls received treatment as usual, and in 10 studies they received another active therapy (spa, hot packs, structured education, supervised relaxation, cognitive behavioural therapy, muscle strengthening, stretching). In six studies an attention control was used (nonstructured education, supervised recreational therapies, transcutaneous electrical neurostimulation or pharmacological placebo) (see Additional file 1).

Three studies compared different intensities of landbased $\mathrm{AE}$, and four studies compared water-based $\mathrm{AE}$ with land-based AE (see Additional file 2).

A total $694 / 889$ (78.1\%) of the patients in the AE groups and $617 / 742(83.1 \%)$ in the control groups completed therapy $(z=-0.3, P=0.7)$.

Fourteen studies performed follow-ups. The median of the latest follow-up was 26 (12 to 208) weeks. Five studies reported that the patients were motivated to continue exercise $[51,56,70,71,75]$. One study recommended no exercise until follow-up evaluation [61]. Two studies assessed the effects of continuing exercise on outcomes $[25,80]$. One study compared the outcomes of continuers of exercise versus noncontinuers at follow-up without mentioning whether continuing exercise had been recommended [80]. Two studies performed an uncontrolled follow-up $[37,60]$.

\section{Outcomes}

There was a great variety of most outcomes measures (see Additional files 1, 2 and 3). Eleven studies reported on side effects. Five studies reported that no side effects occurred, and six studies reported an increase of symptoms leading to a drop out in some cases. Only six patients assigned to $\mathrm{AE}$ were designated to have an adverse event possibly related to exercise (metatarsal stress fracture, plantar fasciitis, ischialgia, transient knee pain).

\section{Risk of bias within studies}

Only two studies fulfilled all predefined criteria of internal and external validity (see Table 1).

\section{Results of individual studies}

The means, SDs, sample sizes and effect estimates of each study can be seen in the forest plots (see Additional files $4,5,6,7,8,9,10,11,12$ and 13).

\section{Synthesis of results}

Aerobic exercise patients versus controls

Data are reported as the SMD (95\% confidence interval). 


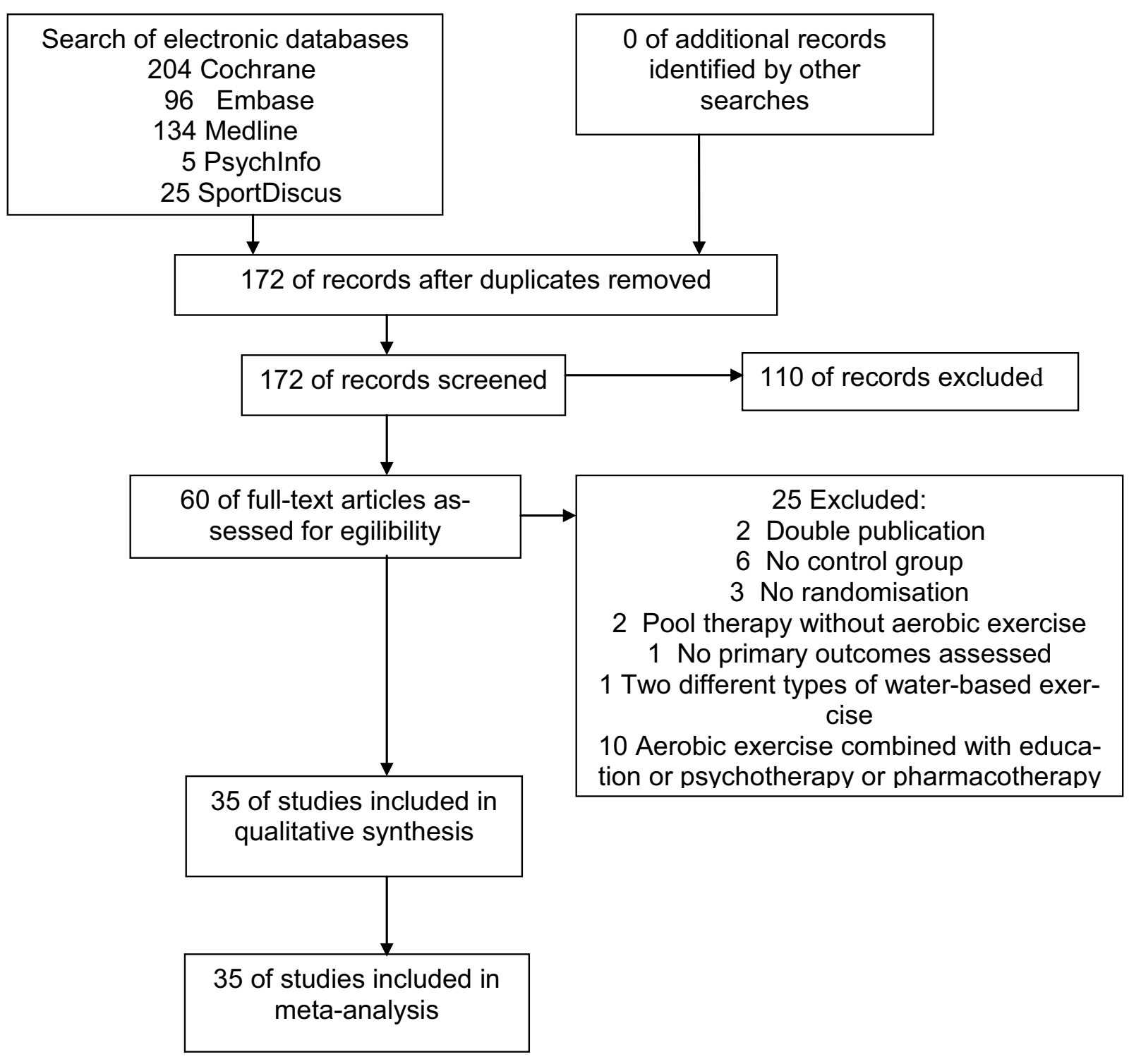

Figure 1 Preferred Reporting Items for Systematic Reviews and Meta-Analyses flow chart. Schematic description of the results of the literature search.

At post treatment, AE reduced pain $(-0.31(-0.46,-$ $0.17) ; P<0.001)$, fatigue $(-0.22(-0.38,-0.05) ; P=0.006)$, depressed mood $(-0.32(-0.53,-0.12) ; P=0.002)$ and limitations of HRQOL $(-0.40(-0.60,-0.20) ; P<0.001)$, and improved physical fitness $(0.65(0.38,0.93) ; P<0.001)$, compared with controls. The effect on sleep $(0.01(-0.19$, $0.21) ; P=0.92)$ was not significant. Based on Cohen's categories, the effects were small for pain, fatigue, depression and HRQOL, and were medium for physical fitness (see Table 4).

At latest follow-up, AE reduced depressed mood (-0.44 $(-0.88,0.01) ; P=0.05)$ and limitations of HRQOL $(-0.27(-$ $0.48,-0.05) ; P=0.01)$, and improved physical fitness $(0.65$
(0.35, 0.96); $P<0.001)$, compared with controls. The effects were small for depressed mood and HRQOL, and were medium for physical fitness. The effects on pain ($0.13(-0.80,0.54) ; P=0.08)$, fatigue $(-0.23(-0.62,0.17) ; P=$ $0.26)$ and sleep $(0.17(-0.14,0.47) ; P=0.26)$ were not significant (see Table 4).

\section{Land-based versus water-based aerobic exercise}

There were no significant effects of water-based AE versus land-based AE on the outcomes pain and depressed mood at post treatment (see Table 2).

Moderate-intensity versus low-intensity aerobic exercise

There were no significant effects of moderate-intensity compared with low-intensity AE on the outcomes pain, 
Table 4: Subgroup analysis for the effect size on pain at post treatment

\begin{tabular}{|c|c|c|c|c|c|}
\hline Outcome title & $\begin{array}{l}\text { Number of } \\
\text { study arms }\end{array}$ & $\begin{array}{l}\text { Number of } \\
\text { patients on AE }\end{array}$ & Effect size $^{a}$ & $\begin{array}{l}\text { Test for overall } \\
\text { effect, } P \text { value }\end{array}$ & $\begin{array}{l}\text { Heterogeneity, I2 } \\
\tau 2 \text { (\%) }\end{array}$ \\
\hline \multicolumn{6}{|l|}{ Type of exercise } \\
\hline Land-based & 22 & 463 & $-0.29(-0.46,-0.13)$ & 0.0005 & $27 ; 0.03$ \\
\hline Water-based & 3 & 61 & $-0.67(-1.04,-0.29)$ & 0.0005 & $0 ; 0$ \\
\hline Mixed & 4 & 43 & $-0.03(-0.45,0.39)$ & 0.89 & $0 ; 0$ \\
\hline \multicolumn{6}{|l|}{ Type of exercise } \\
\hline AE only & 12 & 273 & $-0.35(-0.61,-0.09)$ & 0.0008 & $48 ; 0.09$ \\
\hline $\begin{array}{l}\text { AE combined } \\
\text { with other } \\
\text { exercise }\end{array}$ & 17 & 294 & $-0.28(-0.45,-0.15)$ & 0.001 & $0 ; 0$ \\
\hline \multicolumn{6}{|l|}{ Duration of study } \\
\hline$<7$ weeks & 2 & 32 & $-1.16(-1.86,-0.48)$ & 0.001 & $36 ; 0.09$ \\
\hline 7 to 12 weeks & 13 & 194 & $-0.24(-0.50,-0.02)$ & 0.03 & $16 ; 0.02$ \\
\hline$>12$ weeks & 12 & 338 & $-0.24(-0.40,-0.08)$ & 0.004 & $0 ; 0$ \\
\hline \multicolumn{6}{|l|}{$\begin{array}{l}\text { Frequency of } \\
\text { training/week }\end{array}$} \\
\hline 1 time/week & 2 & 37 & $-0.07(-0.54,03.9)$ & 0.48 & Not applicable \\
\hline 2 times/week & 5 & 127 & $-0.69(-0.95,-0.27)$ & 0.0004 & $35 ; 0.06$ \\
\hline 3 times/week & 16 & 241 & $-0.35(-0.62,-0.09)$ & 0.009 & $48 ; 0.10$ \\
\hline $\begin{array}{l}>3 \text { times/ } \\
\text { week }\end{array}$ & 4 & 142 & $-0.13(-0.38,0.13)$ & 0.33 & $2 ; 0$ \\
\hline \multicolumn{6}{|l|}{$\begin{array}{l}\text { Total duration } \\
\text { aerobic exercise }^{b}\end{array}$} \\
\hline $\begin{array}{l}<1,000 \\
\text { minutes }\end{array}$ & 10 & 175 & $-0.47(-0.86,-0.08)$ & 0.02 & $62 ; 0.19$ \\
\hline $\begin{array}{l}1,000 \text { to } \\
2,000 \\
\text { minutes }\end{array}$ & 9 & 175 & $-0.36(-0.59,-0.13)$ & 0.002 & $0 ; 0$ \\
\hline $\begin{array}{l}>2,000 \\
\text { minutes }\end{array}$ & 8 & 217 & $-0.15(-0.34,0.05)$ & 0.15 & $0 ; 0$ \\
\hline \multicolumn{6}{|l|}{ Intensity of $A E^{c}$} \\
\hline$<50 \%$ maxHR & 1 & 37 & $-0.09(-0.54,0.36)$ & Not applicable & Not applicable \\
\hline $\begin{array}{l}\text { Left up to } \\
\text { patient }\end{array}$ & 2 & 79 & $-0.42(-0.77,-0.07)$ & 0.02 & $0 ; 0$ \\
\hline $\begin{array}{l}>50 \% \\
\operatorname{maxHR}\end{array}$ & 21 & 367 & $-0.26(-0.42,-0.11)$ & 0.0007 & $0 ; 0$ \\
\hline \multicolumn{6}{|l|}{$\begin{array}{l}\text { Type of control } \\
\text { group }\end{array}$} \\
\hline $\begin{array}{l}\text { Attention } \\
\text { placebo }\end{array}$ & 7 & 229 & $-0.27(-0.62,0.07)$ & 0.12 & $67 ; 0.12$ \\
\hline $\begin{array}{l}\text { Therapy as } \\
\text { usual }\end{array}$ & 10 & 147 & $-0.47(-0.71,-0.24)$ & $<0.0001$ & $0 ; 0$ \\
\hline $\begin{array}{l}\text { Active } \\
\text { therapy }\end{array}$ & 10 & 191 & $-0.27(-0.49,-0.06)$ & 0.01 & $0 ; 0$ \\
\hline
\end{tabular}

$\mathrm{AE}$, aerobic exercise; maxHR, maximum of age-adjusted maximum heart rate. aStandardised mean difference ( $95 \%$ confidence interval). $\mathrm{b}$ lf no precise duration of AE was given, $50 \%$ of the total exercise time was assumed for aerobic exercise. cStudies that did not report the intensity of training were excluded from analysis. 
depressed mood and physical fitness at post treatment (see Table 3).

\section{Effects of continuing exercise}

One study found that continuers of exercise at follow-up reported less pain and depression than those who did not exercise [80]. One study found that exercising at followup was related to improvements in physical function and mood [37]. One study reported that pain returned close to the pretraining level during the subsequent de-training [61].

\section{Risk of bias across studies}

There was only substantial heterogeneity in the comparisons of depressed mood and HRQOL at post treatment and for depressed mood at latest follow-up (see Table 2). On visual inspection, the funnel plots of the outcomes post treatment were symmetrical and were thus not indicative for a publication bias (see Additional file 14). Studies with small sample sizes had no significant effect on pain at post treatment (see Table 5).

\section{Additional analyses Subgroup analysis}

Subgroup analyses according to the types of AE, frequency, total time and intensity of $\mathrm{AE}$ and type of control groups did not change the significant effect of $\mathrm{AE}$ on pain at post treatment, except for a combination of waterbased and land-based $\mathrm{AE}$, total duration of $\mathrm{AE}>2,000$ minutes, frequency of training 1 or $>3$ times/week and intensity $<50 \%$ maxHR and attention placebo as control. Statistical heterogeneity of analysis for the effect size for pain was substantially increased in the case of a total duration of $\mathrm{AE}<1,000$ minutes and attention placebo as control (see Table 4).

\section{Sensitivity analysis}

Sensitivity analyses according to potential risks of bias for the outcome pain at post treatment did not change the significant effect of AE on pain at post treatment, except for studies with sample size $<25$ and with only median of outcomes available. Statistical heterogeneity of analysis for the effect size for pain was substantially increased in the case of studies that included patients with mental disorders and with only the median of outcomes available (see Table 5).

\section{Discussion}

\section{Summary of evidence}

$\mathrm{AE}$ reduces pain, fatigue and depressed mood, and improves HRQOL and physical fitness, at post treatment. Positive effects of AE on depressed mood, HRQOL and physical fitness can be detected at latest follow-up. AE has no positive effect on sleep at post treatment, and on pain, fatigue and sleep at follow-up. Continuing exercise is necessary to maintain positive effects on pain.
The following statements are valid for pain reduction at post treatment. There is no evidence of a superiority of water-based over land-based exercise. AE with a slight to moderate intensity is effective. Low-intensity $\mathrm{AE}(<50 \%$ $\operatorname{maxHR}$ ) is not effective. A frequency of AE of 2 to 3 times/week for at least 4 to 6 weeks is necessary for a reduction of symptoms. Combining $\mathrm{AE}$ with stretching or strengthening is no more effective than $\mathrm{AE}$ alone.

The evidence is applicable to the majority of patients in clinical practice except patients with internal and orthopaedic diseases that may prevent $\mathrm{AE}$ and male patients.

\section{Limitations}

Although every effort was made to obtain missing data (outcomes, study design) from the trial authors, it was not possible in every case to obtain these data; the included studies are therefore not represented fully in the meta-analyses. Only medians were available for three studies, but excluding these studies from analysis did not change the results.

The exercise protocol was insufficiently reported by some trials. The positive effects of the training can therefore possibly be attributed to other forms of exercise such as strength, stretching or relaxation, or in the case of pool-based exercise to the effects of warm water. Subgroup analyses did not, however, show a superiority of mixed exercise versus aerobic exercise nor a superiority of pool-based exercise versus land-based exercise.

The prescribed training intensity was either not assessed by heart rate telemetry or was not reported. No definitive conclusions on an effective intensity of $\mathrm{AE}$ are therefore possible.

The attendance rates during the study were inconsistently reported. If continuation of exercise until follow-up was recommended was inconsistently reported too. A subgroup analysis of studies with and without recommended exercise at follow-up was thus not possible.

Side effects were inconsistently reported. No definitive statement on the safety of AE in FMS is therefore possible.

The methodological quality of the studies varied. The positive effect on pain, however, was robust against potential methodological biases.

Given that formal blinding of participants and clinicians to the treatment arm is not possible in trials of exercise, we could have underestimated the extent to which clinicians' and participants' knowledge of group assignation influenced the true effect.

Males and adolescents were rarely included in the study populations. As no gender comparisons were reported, the evidence for the efficacy of AE in men and adolescents with FMS is limited. 
Table 5: Sensitivity analysis for the effect size on pain at post treatment

\begin{tabular}{|c|c|c|c|c|c|}
\hline Outcome title & $\begin{array}{l}\text { Number of } \\
\text { study arms }\end{array}$ & $\begin{array}{l}\text { Number of } \\
\text { patients on } \\
\text { aerobic exercise }\end{array}$ & Effect size $^{a}$ & $\begin{array}{l}\text { Test for overall } \\
\text { effect, } P \text { value }\end{array}$ & $\begin{array}{l}\text { Heterogeneity, I2; } \\
\tau 2(\%)\end{array}$ \\
\hline \multicolumn{6}{|l|}{$\begin{array}{l}\text { Adequate } \\
\text { sequence } \\
\text { generation }\end{array}$} \\
\hline Adequate & 11 & 251 & $-0.20(-0.38,-0.01)$ & 0.04 & $0 ; 0$ \\
\hline $\begin{array}{l}\text { Unclear or } \\
\text { nonadequate }\end{array}$ & 18 & 348 & $-0.39(-0.61,-0.18)$ & 0.0004 & $39 ; 0.07$ \\
\hline \multicolumn{6}{|l|}{$\begin{array}{l}\text { Allocation } \\
\text { concealment }\end{array}$} \\
\hline Adequate & 8 & 223 & $-0.24(-0.47,-0.01)$ & 0.04 & $24 ; 0.02$ \\
\hline $\begin{array}{l}\text { Unclear or } \\
\text { nonadequate }\end{array}$ & 19 & 344 & $-0.35(-0.54,-0.16)$ & 0.0002 & $28 ; 0.04$ \\
\hline \multicolumn{6}{|l|}{$\begin{array}{l}\text { Blinding of } \\
\text { assessor }\end{array}$} \\
\hline Yes & 12 & 306 & $-0.20(-0.36,-0.03)$ & 0.02 & $0 ; 0$ \\
\hline No or unclear & 15 & 261 & $-0.41(-0.66,-0.16)$ & 0.001 & $38 ; 0.08$ \\
\hline \multicolumn{6}{|l|}{ ITT analysis } \\
\hline Yes & 13 & 315 & $-0.22(-0.39,-0.06)$ & 0.009 & $0 ; 0$ \\
\hline No & 14 & 252 & $-0.39(-0.62,-0.16)$ & 0.001 & $36 ; 0$ \\
\hline \multicolumn{6}{|l|}{$\begin{array}{l}\text { Adequacy of } \\
\text { outcomes for } \\
\text { meta-analysis }\end{array}$} \\
\hline Yes (means) & 24 & 517 & $-0.35(-0.51,-0.19)$ & $<0.0001$ & $28 ; 0.04$ \\
\hline No (medians) & 3 & 43 & $-0.05\{-0.43,-0.32)$ & 0.78 & $0 ; 0$ \\
\hline \multicolumn{6}{|l|}{ Sample size } \\
\hline$<25$ & 3 & 30 & $-0.33(-1.00,0.33)$ & 0.33 & $34 ; 0.12$ \\
\hline 25 to 50 & 15 & 188 & $-0.41(-0.70,-0.13)$ & 0.005 & $46 ; 0.11$ \\
\hline$>50$ & 9 & 349 & $-0.23(-0.39,-0.08)$ & 0.004 & $0 ; 0$ \\
\hline \multicolumn{6}{|l|}{$\begin{array}{l}\text { Patients with } \\
\text { mental disorders } \\
\text { included }\end{array}$} \\
\hline Yes & 16 & 312 & $-0.43(-0.78,-0.08)$ & 0.02 & $73 ; 0.28$ \\
\hline No or unclear & 14 & 315 & $-0.40(-0.61,-0.19)$ & 0.0002 & $38 ; 0.05$ \\
\hline
\end{tabular}

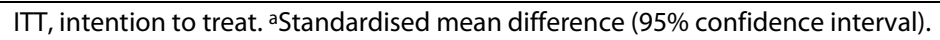

\section{Agreements and disagreements with other systematic reviews}

Our meta-analysis does not confirm the conclusion of a qualitative systematic review that the greatest effects occurred in exercise programmes that were of lower intensity than those of higher intensity [13]. Our data that $\mathrm{AE}$ reduces pain at post treatment are in line with the conclusion of the meta-analyses of the Ottawa Panel [13] and are in contrast to that of the Cochrane review [12]. Not only moderate-intensity AE as recommended by the American Pain Society [5], but also low-intensity AE seems to be effective in reducing pain.

\section{Conclusions}

Implications for clinical practice

The amount and intensity of initial AE should be adapted to the individual level of physical fitness. Patients should start at levels just below their capacity and gradually increase the duration and intensity until they are exercising with low to moderate intensity for 20 to 30 minutes 2 to 3 times/week [12]. It does not seem necessary to assess the heart rate during AE to find the optimum intensity. Patients should exercise with an intensity at which they are able to speak fluently with another person [17]. The choice of the type of AE should be left to the patient's 
preferences and comorbidities and to the local offers of $\mathrm{AE}$ [11]. A training programme should last a minimum of 4 weeks. Patients should be educated that they may have some tolerable short-term increases in pain and fatigue but, if they exercise at an appropriate intensity, these symptoms should return to baseline levels within the first few weeks of exercise [12,17]. Patients should be motivated to continue exercise if they perceive a reduction of symptoms after the programme.

Because AE does not reduce sleeping disturbances, a combination of $\mathrm{AE}$ with medication effective for improving sleep - for example, tricyclic or dual antidepressants or pregabalin $[81,82]$ - should be considered.

\section{Implications for research}

Four main questions need to be answered by future studies. By which methods (for example, education, booster sessions) can patients be motivated to continue exercise? Is aerobic and mixed exercise cost-effective [83]? Is the combination of AE with pharmacological therapy superior to $\mathrm{AE}$ or medication alone? Which sociodemographic and clinical variables predict a positive and negative treatment outcome?

Future studies on these topics should focus on larger sample sizes (multicentre studies including a sufficient number of men and adolescents and patients with mental and somatic comorbidities). Study quality could be improved by detailed reporting of demographic and clinical data of the study groups at baseline, exercise protocol and adherence to interventions (attendance rates, adherence to prescribed intensity assessed by heart rate telemetry), creation of a standardised protocol to report adverse events and use of standard outcome measures.

\section{Additional material}

Additional file 1 Search strategy for MEDLINE. The file contains the literature search strategy for the database MEDLINE.

Additional file $\mathbf{2}$ Main characteristics of studies with aerobic and mixed exercise in fibromyalgia syndrome. The file contains the main characteristics of studies with aerobic and mixed exercise in fibromyalgia syndrome including outcomes measures.

Additional file 3 Main characteristics of studies with head to head comparisons of different types of aerobic and mixed exercise in fibromyalgia syndrome. The file contains the main characteristics of studies with head-to-head comparisons of different types of aerobic and mixed exercise in fibromyalgia syndrome including outcome measures.

Additional file 4 Effect estimates (standardised mean differences) of aerobic exercise versus controls on pain at post treatment. Forest plots show standardised mean differences (effect sizes) from the random effects model (inverse variance method). A negative effect indicates that the endpoint score of the outcome in the exercise groups is lower than in control group in the study. The pooled (all studies together) effect size is weighted by the inverse variance of each study. IV, inverse variance (method); SD, standard deviation; Std. mean difference, standardised mean differences; random, random effects model; SD, standard deviation; total, number of patients; weight, relative weight (\%) of the study in the calculation.
Additional file 5 Effect estimates (standardised mean differences) of aerobic exercise versus controls on fatigue and sleep at post treatment. Forest plots show standardised mean differences (effect sizes) from the random effects model (inverse variance method). A negative effect indicates that the endpoint score of the outcome in the exercise groups is

lower than in control group in the study. The pooled (all studies together) effect size is weighted by the inverse variance of each study. IV, inverse variance (method); SD, standard deviation; Std. mean difference, standardised mean differences; random, random effects model; SD, standard deviation; total, number of patients; weight, relative weight (\%) of the study in the calculation.

Additional file 6 Effect estimates (standardised mean differences) of aerobic exercise versus controls on depressed mood at post treat-

ment. Forest plots show standardised mean differences (effect sizes) from the random effects model (inverse variance method). A negative effect indicates that the endpoint score of the outcome in the exercise groups is lower than in control group in the study. The pooled (all studies together) effect size is weighted by the inverse variance of each study. IV, inverse variance (method); SD, standard deviation; Std. mean difference, standardised mean differences; random, random effects model; SD, standard deviation; total, number of patients; weight, relative weight (\%) of the study in the calculation.

Additional file 7 Effect estimates (standardised mean differences) of aerobic exercise versus controls on quality of life at post treatment. Forest plots show standardised mean differences (effect sizes) from the random effects model (inverse variance method). A negative effect indicates that the endpoint score of the outcome in the exercise groups is lower than in control group in the study. The pooled (all studies together) effect size is weighted by the inverse variance of each study. IV, inverse variance (method); SD, standard deviation; Std. mean difference, standardised mean differences; random, random effects model; SD, standard deviation; total, number of patients; weight, relative weight (\%) of the study in the calculation.

Additional file 8 Effect estimates (standardised mean differences) of aerobic exercise versus controls on physical fitness at post treatment. Forest plots show standardised mean differences (effect sizes) from the random effects model (inverse variance method). A negative effect indicates that the endpoint score of the outcome in the exercise groups is lower than in control group in the study. The pooled (all studies together) effect size is weighted by the inverse variance of each study. IV, inverse variance (method); SD, standard deviation; Std. mean difference, standardised mean differences; random, random effects model; SD, standard deviation; total, number of patients; weight, relative weight (\%) of the study in the calculation.

Additional file 9 Effect estimates (standardised mean differences) of aerobic exercise versus controls on pain and fatigue at latest followup. Forest plots show standardised mean differences (effect sizes) from the random effects model (inverse variance method). A negative effect indicates that the endpoint score of the outcome in the exercise groups is lower than in control group in the study. The pooled (all studies together) effect size is weighted by the inverse variance of each study. IV, inverse variance (method); SD, standard deviation; Std. mean difference, standardised mean differences; random, random effects model; SD, standard deviation; total, number of patients; weight, relative weight (\%) of the study in the calculation.

Additional file 10 Effect estimates (standardised mean differences) of aerobic exercise versus controls on sleep and depressed mood at latest follow-up. Forest plots show standardised mean differences (effect sizes) from the random effects model (inverse variance method). A negative effect indicates that the endpoint score of the outcome in the exercise groups is lower than in control group in the study. The pooled (all studies together) effect size is weighted by the inverse variance of each study. IV, inverse variance (method); SD, standard deviation; Std. mean difference, standardised mean differences; random, random effects model; SD, standard deviation; total, number of patients; weight, relative weight (\%) of the study in the calculation. 


\begin{abstract}
Additional file 11 Effect estimates (standardised mean differences) of aerobic exercise versus controls on quality of life and physical fitness at latest follow-up. Forest plots show standardised mean differences (effect sizes) from the random effects model (inverse variance method). A negative effect indicates that the endpoint score of the outcome in the exercise groups is lower than in control group in the study. The pooled (all studies together) effect size is weighted by the inverse variance of each study. IV, inverse variance (method); SD, standard deviation; Std. mean difference, standardised mean differences; random, random effects model; SD, standard deviation; total, number of patients; weight, relative weight (\%) of the study in the calculation.
\end{abstract}

Additional file 12 Effect estimates (standardised mean differences) of moderate versus low intensity on pain and depressed mood post treatment. Forest plots show standardised mean differences (effect sizes) from the random effects model (inverse variance method). A negative effect indicates that the endpoint score of the outcome in the exercise group with moderate intensity is lower than in exercise group with low intensity. The pooled (all studies together) effect size is weighted by the inverse variance of each study. IV, inverse variance (method); SD, standard deviation; Std. mean difference, standardised mean differences; random, random effects model; SD, standard deviation; total, number of patients; weight, relative weight (\%) of the study in the calculation.

Additional file 13 Effect estimates (standardised mean differences) of water versus land-based aerobic exercise on pain and depressed mood post treatment. Forest plots show standardised mean differences (effect sizes) from the random effects model (inverse variance method). A negative effect indicates that the endpoint score of the outcome in the water-based exercise groups is lower than in the land-based exercise group in the study. The pooled (all studies together) effect size is weighted by the inverse variance of each study. IV, inverse variance (method); SD, standard deviation; Std. mean difference, standardised mean differences; random, random effects model; SD, standard deviation; total, number of patients; weight, relative weight (\%) of the study in the calculation.

Additional file 14 Funnel plot of the comparisons of aerobic exercise versus controls on pain. Scatter plot of the intervention effect estimates (standardised mean differences (SMD)) from individual studies against their standard errors (SE) (on a reversed scale). Publication bias may lead to asymmetry in funnel plots on visual inspection.

\section{Abbreviations}

AE: aerobic and mixed exercise; FMS: fibromyalgia syndrome; HRQOL: healthrelated quality of life; maxHR: maximum heart rate; $\mathrm{RCT}$ : randomised controlled trial; SD: standard deviation; SMD: standardised mean difference.

\section{Competing interests}

WH received honoraria for educational lectures from Eli Lilly, Janssen-Cilag and Mundipharma, consulting fees from Eli-Lilly and Pfizer, and a congress travel grant from Eli-Lilly. MSc received consulting honoraria from Pfizer and MSD. None of these organisations financed this manuscript (including the articleprocessing charge). The other authors declare that they have no competing interests.

\section{Authors' contributions}

WH conceived the hypothesis of the manuscript, participated in the data collection, conducted the statistical analysis, wrote the first draft of the manuscript and had primary responsibility for the manuscript. PK, JL, BM, MSt, MSc and $A B$ participated in the collection of the data and analysis of the studies (see Materials and methods). AB and MSc participated in the study design and the interpretation of the data. All authors critically reviewed, contributed and approved the final manuscript.

\section{Authors' information}

WH and MSc are vice-presidents of the German Interdisciplinary Association of Pain Therapy DIVS and were responsible for the development on the German interdisciplinary guideline on the classification, pathophysiology and management of FMS. AB is head of the Cochrane group on fibromyalgia.

\section{Author Details}

1 Department of Internal Medicine I, Klinikum Saarbrücken, Winterberg 1, D66119 Saarbrücken, Germany, ${ }^{2}$ Department of Psychosomatic Medicine, Technische Universität München, Langestr. 3, D-81675 München, Germany, ${ }^{3}$ Department of Internal Medicine V (Integrative Medicine), University of Duisburg-Essen, Kliniken Essen-Mitte, Am Deimelsberg 34a, D-45276 Essen, Germany, ${ }^{4}$ Orthopaedic Clinic, University of Heidelberg, Schlierbacher Landstraße 200, D-69118 Heidelberg, Germany and 5School of Physical Therapy, University of Saskatchewan, Saskatoon, 1121 College Drive, Saskatoon SK S7N OW3, Canada

\section{Received: 4 November 2009 Revised: 8 February 2010}

Accepted: 10 May 2010 Published: 10 May 2010

\section{References}

1. Wolfe F, Smythe HA, Yunus MB, Bennett RM, Bombardier C, Goldenberg DL, Tugwell P, Campbell SM, Abeles M, Clark P, Fam AG, Farber SJ, Fiechtner JJ, Franklin CM, Gatter RA, Hamaty D, Lessard J, Lichtbroun AS, Masi AT, McCain GA, Reynolds WJ, Romano TJ, Russel IJ, Sheon RP: The American College of Rheumatology 1990 criteria for the classification of fibromyalgia: report of the multicenter criteria committee. Arthritis Rheum 1990, 33:160-172.

2. Branco JC, Bannwarth B, Failde I, Abello J Carbonell, Blotman F, Spaeth M, Saraiva F, Nacci F, Thomas E, Caubère JP, Le Lay K, Taieb C, Matucci-Cerinic $M$ : Prevalence of fibromyalgia: a survey in five European countries. Semin Arthritis Rheum 2009 in press.

3. Henningsen $\mathrm{P}$, Zimmermann $\mathrm{T}$, Sattel H: Medically unexplained physical symptoms, anxiety, and depression: a meta-analytic review. Psychosom Med 2003, 65:528-533.

4. Robinson $\mathrm{RL}$, Jones $\mathrm{ML}$ : In search of pharmacoeconomic evaluations for fibromyalgia treatments: a review. Expert Opin Pharmacother 2006, 7:1027-1039.

5. Burckhardt CS, Goldenberg D, Crofford L, Gerwin R, Gowans S, Jackson K, Kugel P, McCarberg W, Ruidn N, Schnaberg L, Taylor AG, Taylor J, Turk D: Guideline for the Management of Fibromyalgia Syndrome. Pain in Adults and Children APS Clinical Practice Guideline Series No. 4. Glenview, IL: American Pain Society; 2005

6. Häuser W, Arnold B, Eich W, Felde E, Flügge C, Henningsen P, Herrmann $M$, Köllner $V$, Kühn E, Nutzinger D, Offenbächer $M$, Schiltenwolf $M$, Sommer C, Thieme K, Kopp I: Management of fibromyalgia syndrome an interdisciplinary evidence-based guideline. Ger Med Sci 2008, 6:Doc14.

7. Carville SF, Arendt-Nielsen S, Bliddal H, Blotman F, Branco JC, Buskila D, Da Silva JA, Danneskiold-Samsøe B, Dincer F, Henriksson C, Henriksson KG, Kosek E, Longley K, McCarthy GM, Perrot S, Puszczewicz M, Sarzi-Puttini P, Silman A, Späth M, Choy EH, European League Against Rheumatism: EULAR evidence based recommendations for the management of fibromyalgia syndrome. Ann Rheum Dis 2008, 67:536-541.

8. van Koulil S, Effting M, Kraaimaat FW, van Lankveld W, van Helmond T, Cats $\mathrm{H}$, van Riel PL, de Jong AJ, Haverman JF, Evers AW: Cognitivebehavioural therapies and exercise programmes for patients with fibromyalgia: state of the art and future directions. Ann Rheum Dis 2007, 66:571-581.

9. Schiltenwolf M, Häuser W, Felde E, Flügge C, Häfner R, Settan M, Offenbächer M: Physiotherapy, exercise and strength training and physical therapies in the treatment of fibromyalgia syndrome. Schmerz 2008, 22:303-312. [in German]

10. Gowans SE, de Hueck A: Pool exercise for individuals with fibromyalgia. Curr Opin Rheumatol 2007, 19:168-173.

11. Jones KD, Lipton G: Exercise interventions in fibromyalgia: clinical applications from the evidence. Rheum Dis Clin North Am 2009, 35:373-391.

12. Busch AJ, Barber KA, Overend TJ, Peloso PM, Schachter CL: Exercise for treating fibromyalgia syndrome. Cochrane Database Syst Rev 2007:CD003786

13. Brosseau L, Wells GA, Tugwell P, Egan M, Wilson KG, Dubouloz CJ, Casimiro L, Robinson VA, McGowan J, Busch A, Poitras S, Moldofsky H, Harth M, Finestone HM, Nielson W, Haines-Wangda A, Russell-Doreleyers M, Lambert K, Marshall AD, Veilleux L, Ottawa Panel Members: Ottawa Panel 
evidence-based clinical practice guidelines for aerobic fitness exercise in the management of fibromyalgia: part 1. Phys Ther 2008, 88:857-871.

14. Moher D, Liberati A, Tetzlaff J, Altman DG, PRISMA Group: Preferred Reporting Items for Systematic Reviews and Meta-Analyses: the PRISMA statement. Ann Intern Med 2009, 151:264-269.

15. Cochrane handbook for systematic reviews of intervention. Version 5.01 [http://www.cochrane.org/resources/handbook/handbook.pdf

16. Häuser W, Bernardy K, Arnold B, Offenbächer M, Schiltenwolf M: Efficacy of multicomponent treatment in fibromyalgia syndrome: a metaanalysis of randomized controlled clinical trials. Arthritis Rheum 2009, 61:216-224.

17. Rooks DS: Talking to patients with fibromyalgia about physical activity and exercise. Curr Opin Rheumatol 2008, 20:208-212.

18. The Nordic Cochrane Centre: Review Manager (RevMan) [computer program]. Version 5.0 for Windows. Copenhagen: The Cochrane Collaboration; 2009

19. Laird NM, Mosteller F: Some statistical methods for combining experimental results. Int J Technol Assess Health Care 1999, 6:5-30

20. Cohen J: Statistical Power Analysis for the Behavoral Sciences Hillsdale, NJ: Lawrence Erlbaum Associates; 1988.

21. Tomas-Carus P, Häkkinen A, Gusi N, Leal A, Häkkinen K, Ortega-Alonso A: Aquatic training and detraining on fitness and quality of life in fibromyalgia. Med Sci Sports Exerc 2007, 39:1044-1050.

22. Munguía-Izquierdo $D$, Legaz-Arrese $A$ : Exercise in warm water decreases pain and improves cognitive function in middle-aged women with fibromyalgia. Clin Exp Rheumatol 2007, 25:823-830.

23. Dobkin PL, Abrahamowicz M, Fitzcharles MA, Dritsa M, da Costa D: Maintenance of exercise in women with fibromyalgia. Arthritis Rheum 2005, 53:724-731

24. Dobkin PL, Da Costa D, Abrahamowicz M, Dritsa M, Du Berger R, Fitzcharles MA, Lowensteyn I: Adherence during an individualized home based 12-week exercise program in women with fibromyalgia. J Rheumatol 2006, 33:333-341.

25. Gowans SE, Dehueck A, Voss S, Silaj A, Abbey SE: Six-month and one-year followup of 23 weeks of aerobic exercise for individuals with fibromyalgia. Arthritis Rheum 2004, 51:890-898.

26. Mannerkorpi K, Ahlmen M, Ekdahl C: Six- and 24-month follow-up of pool exercise therapy and education for patients with fibromyalgia. Scand J Rheumatol 2002, 31:306-310.

27. Rooks DS, Silverman CB, Kantrowitz FG: The effects of progressive strength training and aerobic exercise on muscle strength and cardiovascular fitness in women with fibromyalgia: a pilot study. Arthritis Rheum 2002, 47:22-28.

28. Dawson KA, Tiidus PM, Pierreynowski M, Crawford JP, Trotter J: Evaluation of a community-based exercise program for diminishing symptoms of fibromyalgia. Physiother Canada 2003, 55:17-22.

29. Gandhi N, DePauw KP, Dolny DG, Freson T: Effect of an exercise program on quality of life of women with fibromyalgia. Women Ther 2002, 25:91-103.

30. Meiworm L, Jakob E, Walker UA, Peter HH, Keul J: Patients with fibromyalgia benefit from aerobic endurance exercise. Clin Rheumatol 2000, 19:253-257.

31. Salek AK, Khan MM, Ahmed SM, Rashid MI, Emran MA, Mamun MA: Effect of aerobic exercise on patients with primary fibromyalgia syndrome. Mymensingh Med J 2005, 14:141-144.

32. Mannerkorpi K, Nyberg B, Ahlmen M, Ekdahl C: Pool exercise combined with an education program for patients with fibromyalgia syndrome. A prospective, randomized study. J Rheumatol 2000, 27:2473-2481.

33. Ide MR, Belini MA, Caromano FA: Effects of an aquatic versus nonaquatic respiratory exercise program on the respiratory muscle strength in healthy aged persons. Clinics (Sao Paulo) 2005, 60:151-158.

34. de Andrade SC, de Carvalho RF, Soares AS, de Abreu Freitas RP, de Medeiros Guerra LM, Vilar MJ: Thalassotherapy for fibromyalgia: a randomized controlled trial comparing aquatic exercises in sea water and water pool. Rheumatol Int 2008, 29:147-152.

35. Verstappen FT, van Santen-Hoeuft HM, Bolwijn S: Effect of a group activity program for fibromyalgia patients on physical fitness and wellbeing. J Musculoskeletal Pain 1997, 5:17-28

36. Burckhardt CS, Mannerkorpi K, Hedenberg L, Bjelle A: A randomized, controlled clinical trial of education and physical training for women with fibromyalgia. J Rheumatol 1994, 21:714-720.
37. Gowans SE, deHueck A, Voss S, Richardson M: A randomized, controlled trial of exercise and education for individuals with fibromyalgia. Arthritis Care Res 1999, 12:120-128.

38. Hammond A, Freeman K: Community patient education and exercise for people with fibromyalgia: a parallel group randomized controlled trial. Clin Rehabil 2006, 20:835-846.

39. Isomeri R, Mikkelson M, Latikka P, Kammonen K: Effects of amitriptyline and cardiovascular fitness training on pain in patients with primary fibromyalgia. J Musculoskeletal Pain 1993, 3/4:253-256.

40. Gustafsson M, Ekholm J, Broman L: Effects of a multiprofessional rehabilitation programme for patients with fibromyalgia syndrome. $J$ Rehabil Med 2002, 34:119-127.

41. Cedraschi C, Desmeules J, Rapiti E, Baumgartner E, Cohen P, Finckh A, Allaz AF, Vischer TL: Fibromyalgia: a randomised, controlled trial of a treatment programme based on self management. Ann Rheum Dis 2004, 63:290-296.

42. Keel PJ, Bodoky C, Gerhard U, Muller W: Comparison of integrated group therapy and group relaxation training for fibromyalgia. Clin J Pain 1998, 14:232-238.

43. Lemstra M, Olszynski WP: The effectiveness of multidisciplinary rehabilitation in the treatment of fibromyalgia: a randomized controlled trial. Clin J Pain 2005, 21:166-174.

44. Mason LW, Goolkasian P, McCain GA: Evaluation of multimodal treatment program for fibromyalgia. J Behav Med 1998, 21:163-178.

45. ZijJstra TR, Laar MA van de, Bernelot HJ Moens, Taal E, Zakraoui L, Rasker JJ: Spa treatment for primary fibromyalgia syndrome: a combination of thalassotherapy, exercise and patient education improves symptoms and quality of life. Rheumatology (Oxford) 2005, 44:539-546.

46. Meyer BB, Lemley KJ: Utilizing exercise to affect the symptomology of fibromyalgia: a pilot study. Med Sci Sports Exerc 2000, 32:1691-1697.

47. Stephens S, Feldman BM, Bradley N, Schneiderman J, Wright V, SinghGrewal D, Lefebvre A, Benseler SM, Cameron B, Laxer R, O'Brien C, Schneider R, Silverman E, Spiegel L, Stinson J, Tyrrell PN, Whitney K, Tse $S M$ : Feasibility and effectiveness of an aerobic exercise program in children with fibromyalgia: results of a randomized controlled pilot trial. Arthritis Rheum 2008, 59:1399-1406.

48. van Santen M, Bolwijn P, Landewé R, Verstappen F, Bakker C, Hidding A, Kemp D van Der, Houben H, Linden S van der: High or low intensity aerobic fitness training in fibromyalgia: does it matter? J Rheumatol 2002, 29:582-587.

49. Assis MR, Silva LE, Alves AM, Pessanha AP, Valim V, Feldman D, Neto TL, Natour J: A randomized controlled trial of deep water running: clinical effectiveness of aquatic exercise to treat fibromyalgia. Arthritis Rheum 2006, 55:57-65.

50. Evcik D, Yigit I, Pusak H, Kavuncu V: Effectiveness of aquatic therapy in the treatment of fibromyalgia syndrome: a randomized controlled open study. Rheumatol Int 2008, 28:885-890

51. Jentoft ES, Kvalvik AG, Mengshoel AM: Effects of pool-based and landbased aerobic exercise on women with fibromyalgia/chronic widespread muscle pain. Arthritis Rheum 2001, 45:42-47.

52. de Melo Vitorino DF, de Carvalho BC, do Prado GF: Hydrotherapy and conventional physiotherapy improbe sleep time and quality of life of fibromyalgia patients: randomised clinical trial. Sleep Med 2006, 7:293-296.

53. Alentorn-Geli E, Padilla J, Moras G, Lázaro Haro C, Fernández-Solà J: Six weeks of whole-body vibration exercise improves pain and fatigue in women with fibromyalgia. J Altern Complement Med 2008, 14:975-981.

54. Altan L, Bingol U, Aykac M, Koc Z, Yurtkuran M: Investigation of the effects of pool-based exercise on fibromyalgia syndrome. Rheumatol Int 2004, 24:272-277.

55. Bircan C, Karasel SA, Akgün B, El O, Alper S: Effects of muscle strengthening versus aerobic exercise program in fibromyalgia. Rheumatol Int 2008, 28:527-532.

56. Buckelew SP, Conway R, Parker J, Deuser WE, Read J, Witty TE, Hewett JE, Minor M, Johnson JC, Van Male L, McIntosh MJ, Nigh M, Kay DR: Biofeedback/relaxation training and exercise interventions for fibromyalgia: a prospective trial. Arthritis Care Res 1998, 11:196-209.

57. Da Costa D, Abrahamowicz M, Lowensteyn I, Bernatsky S, Dritsa M, Fitzcharles MA, Dobkin PL: A randomized clinical trial of an individualized home-based exercise programe for women with fibromyalgia. Rheumatology (Oxford) 2005, 44:1422-1427. 
58. Etnier JL, Karper WB, Gapin Jl, Barella LA, Chang YK, Murphy KJ: Exercise, fibromyalgia, and fibrofog: a pilot study. J Phys Act Health 2009, 6:239-246.

59. Fontaine KR, Haas S: Effects of lifestyle physical activity on health status, pain, and function in adults with fibromyalgia syndrome. $J$ Musculoskeletal Pain 2007, 15:3-9.

60. Gowans SE, deHueck A, Voss S, Silaj A, Abbey SE, Reynolds WJ: Effect of a randomized, controlled trial of exercise on mood and physical function in individuals with fibromyalgia. Arthritis Rheum 2001, 45:519-529.

61. Gusi N, Tomas-Carus P, Hakkinen A, Hakkinen K, Ortega-Alonso A: Exercise in waist-high warm water decreases pain and improves health-related quality of life and strength in the lower extremities in women with fibromyalgia. Arthritis Rheum 2006, 55:66-73.

62. Jones KD, Burckhardt CS, Deodhar AA, Deodhar AA, Perrin NA, Hanson GC, Bennett RM: A six-month randomized controlled trial of exercise and pyridostigmine in the treatment of fibromyalgia. Arthritis Rheum 2008 58:612-622.

63. King SJ, Wessel J, Bhambhani Y, Sholter D, Maksymowych W: The effects of exercise and education, individually or combined, in women with fibromyalgia. J Rheumato/ 2002, 29:2620-2627.

64. Martin L, Nutting A, Maclntosh BR, Edworthy SM, Butterwick D, Cook J: An exercise program in the treatment of fibromyalgia. J Rheumatol 1996, 23:1050-1053.

65. McCain GA, Bell DA, Mai FM, Halliday PD: A controlled study of the effects of a supervised cardiovascular fitness training program on the manifestations of primary fibromyalgia. Arthritis Rheum 1988, 31:1135-1141

66. Mengshoel AM, Komnaes HB, Forre O: The effects of 20 weeks of physical fitness training in female patients with fibromyalgia. Clin Exp Rheumatol 1992, 10:345-349.

67. Munguía-Izquierdo D, Legaz-Arrese A: Assessment of the effects of aquatic therapy on global symptomatology in patients with fibromyalgia syndrome: a randomized controlled trial. Arch Phys Med Rehabil 2008, 89:2250-2257.

68. Nichols DS, Glenn TM: Effects of aerobic exercise on pain perception affect, and level of disability in individuals with fibromyalgia. Phys Ther 1994, 74:327-332.

69. Norregaard J, Lykkegaard JJ, Mehlsen J, Danneskiold-Samsooe B: Exercise training in treatment of fibromyalgia. J Musculoskelet Pain 1997, 5:71-79.

70. Ramsay C, Moreland J, Ho M, Joyce S, Walker S, Pullar T: An observerblinded comparison of supervised and unsupervised aerobic exercise regimens in fibromyalgia. Rheumatology (Oxford) 2000, 39:501-515.

71. Redondo JR, Justo CM, Moraleda FV, Velayos YG, Puche JJ, Zubero JR, Hernández TG, Ortells LC, Pareja MA: Long-term efficacy of therapy in patients with fibromyalgia: a physical exercise-based program and a cognitive-behavioral approach. Arthritis Rheum 2004, 51:184-192.

72. Richards SC, Scott DL: Prescribed exercise in people with fibromyalgia: parallel group randomised controlled trial. BMJ 2002, 325:185.

73. Rooks DS, Gautam S, Romeling M, Cross ML, Stratigakis D, Evans B, Goldenberg DL, Iversen MD, Katz JN: Group exercise, education, and combination self-management in women with fibromyalgia: a randomized trial. Arch Intern Med 2007, 167:2192-2200.

74. Schachter CL, Busch AJ, Peloso PM, Sheppard MS: Effects of short versus long bouts of aerobic exercise in sedentary women with fibromyalgia: a randomized controlled trial. Phys Ther 2003, 83:340-358.

75. Şencan S, Ak S, Karan A, Müslümanoğlu L, Özcan E, Berker E: A study to compare the therapeutic efficacy of aerobic exercise and paroxetine in fibromyalgia syndrome. J Back Musculoskeletal Rehabil 2004, 17:57-61.

76. Tomas-Carus P, Gusi N, Häkkinen A, Häkkinen K, Leal A, Ortega-Alonso A: Eight months of physical training in warm water improves physical and mental health in women with fibromyalgia: a randomized controlled trial. J Rehabil Med 2008, 40:248-252.

77. Valim V, Oliveira L, Suda A, Silva L, de Assis M, Barros T Neto, Feldman D, Natour J: Aerobic fitness effects in fibromyalgia. J Rheumatol 2003, 30:1060-1069

78. Valkeinen $\mathrm{H}$, Alén M, Häkkinen A, Hannonen P, Kukkonen-Harjula K, Häkkinen K: Effects of concurrent strength and endurance training on physical fitness and symptoms in postmenopausal women with fibromyalgia: a randomized controlled trial. Arch Phys Med Rehabil 2008, 89:1660-1666

79. van Santen $M$, Bolwijn $P$, Verstappen $F$, Bakker $C$, Hidding $A$, Houben $H$, Heijde D van der, Landewé $R$, Linden S van der: A randomized clinical trial comparing fitness and biofeedback training versus basic treatment in patients with fibromyalgia. J Rheumatol 2002, 29:575-581.

80. Wigers SH, Stiles TC, Vogel PA: Effects of aerobic exercise versus stress management treatment in fibromyalgia. A 4.5 year prospective study. Scand J Rheumatol 1996, 25:77-86

81. Häuser W, Bernardy K, Üceyler N, Sommer C: Treatment of fibromyalgia syndrome with antidepressants - a meta-analysis. JAMA 2009, 301:198-209.

82. Häuser W, Bernardy K, Üceyler N, Sommer C: Treatment of fibromyalgia syndrome with gabapentin and pregabalin - a meta-analysis of randomized controlled trails. Pain 2009, 145:69-81.

83. Gusi N, Tomas-Carus P: Cost-utility of an 8-month aquatic training for women with fibromyalgia: a randomized controlled trial. Arthritis Res Ther 2008, 10:R24.

doi: $10.1186 / \operatorname{ar} 3002$

Cite this article as: Häuser et al., Efficacy of different types of aerobic exercise in fibromyalgia syndrome: a systematic review and meta-analysis of randomised controlled trials Arthritis Research \& Therapy 2010, 12:R79

\section{Submit your next manuscript to BioMed Centra and take full advantage of:}

- Convenient online submission

- Thorough peer review

- No space constraints or color figure charges

- Immediate publication on acceptance

- Inclusion in PubMed, CAS, Scopus and Google Scholar

- Research which is freely available for redistribution
C Biomed Central 